\title{
On Schubert calculus in elliptic cohomology
}

\author{
Cristian Lenart $\|^{\prime}$ and Kirill Zainoulline $\|^{2}$ \\ ${ }^{1}$ Department of Mathematics and Statistics, State University of New York at Albany, Albany, NY 12222, USA \\ ${ }^{2}$ Department of Mathematics and Statistics, University of Ottawa, 585 King Edward Street, Ottawa, ON, K1N 6N5, \\ Canada
}

\begin{abstract}
An important combinatorial result in equivariant cohomology and $K$-theory Schubert calculus is represented by the formulas of Billey and Graham-Willems for the localization of Schubert classes at torus fixed points. These formulas work uniformly in all Lie types, and are based on the concept of a root polynomial. We define formal root polynomials associated with an arbitrary formal group law (and thus a generalized cohomology theory). We use these polynomials to simplify the approach of Billey and Graham-Willems, as well as to generalize it to connective $K$-theory and elliptic cohomology. Another result is concerned with defining a Schubert basis in elliptic cohomology (i.e., classes independent of a reduced word), using the Kazhdan-Lusztig basis of the corresponding Hecke algebra.
\end{abstract}

Résumé. Un résultat combinatoire important dans le calcul de Schubert pour la cohomologie et la $K$-théorie équivariante est représenté par les formules de Billey et Graham-Willems pour la localisation des classes de Schubert aux points fixes du tore. Ces formules sont uniformes pour tous les types de Lie, et sont basés sur le concept d'un polynôme de racines. Nous définissons les polynômes formels de racines associées à une loi arbitraire de groupe formel (et donc à une théorie de cohomologie généralisée). Nous utilisons ces polynômes pour simplifier les preuves de Billey et Graham-Willems, et aussi pour généraliser leurs résultats à la $K$-théorie connective et la cohomologie elliptique. Un autre résultat concerne la définition d'une base de Schubert dans cohomologie elliptique (c'est à dire, des classes indépendantes d'un mot réduit), en utilisant la base de Kazhdan-Lusztig de l'algèbre de Hecke correspondant.

Keywords: Schubert classes, Bott-Samelson classes, elliptic cohomology, root polynomial, Kazhdan-Lusztig basis

\section{Introduction}

Modern Schubert calculus has been mostly concerned with the cohomology and $K$-theory (as well as their quantum deformations) of generalized flag manifolds $G / B$, where $G$ is a connected complex semisimple Lie group and $B$ a Borel subgroup; Kac-Moody flag manifolds have also been studied, but we will restrict ourselves here to the finite case. The basic results in Schubert calculus for other cohomology theories have only been obtained recently in [CZZ12, CZZ13, CZZ14, CPZ13, GR13, HHH05, HK11, KK13]. After this main theory has been developed, the next step is to give explicit formulas, thus generalizing

\footnotetext{
${ }^{\dagger}$ Partially supported by the NSF grants DMS-1101264 and DMS-1362627; the hospitality and support of the Max-PlanckInstitut für Mathematik in Bonn, where part of this work was carried out, are also gratefully acknowledged.

$\ddagger$ Partially supported by the NSERC Discovery grant 385795-2010 and the Early Researcher Award (Ontario). 
well-known results in cohomology and $K$-theory, which are usually based on combinatorial structures. This paper is a contribution in this direction; for some of the related proofs, see [LZ14].

We focus on elliptic cohomology, more precisely, on the associated hyperbolic formal group law, which we view as the first case after $K$-theory in terms of complexity. (The correspondence between generalized cohomology theories and formal group laws is explained below.) The main difficulty beyond $K$-theory is the fact that the naturally defined cohomology classes corresponding to a Schubert variety depend on its chosen Bott-Samelson desingularization, and thus on a reduced word for the given Weyl group element (which is not the case in ordinary cohomology and $K$-theory, where we have naturally defined Schubert classes). Thus, one problem is to express combinatorially these so-called Bott-Samelson classes.

In this paper, we extend the combinatorial formulas for localizations of Schubert classes in (torus equivariant) cohomology and $K$-theory, which are due to Billey [Bi99] (cf. also [AJS94]) and Graham-Willems [Gr02, Wil04], respectively. These formulas are based on the concept of a root polynomial. We define formal root polynomials, which correspond to an arbitrary formal group law. We explain the connection of these polynomials to the hyperbolic solution of the Yang-Baxter equation. We then show that, in the hyperbolic case, the formal root polynomials provide an efficient way to compute the transition matrix between two natural bases of the formal Demazure algebra. As a corollary, we derive in a simpler and uniform manner (i.e., in all Lie types, and for both ordinary cohomology and $K$-theory) the connective $K$-theory version of the formulas of Billey and Graham-Willems, which seems new. We also derive a duality result in connective $K$-theory, as well as other related results, and state a positivity conjecture.

Another result is concerned with defining a Schubert basis in elliptic cohomology (i.e., classes independent of a reduced word), using the Kazhdan-Lusztig basis of the corresponding Hecke algebra.

\section{Background}

We briefly recall the main results in Schubert calculus for generalized cohomology theories.

\subsection{Complex oriented cohomology theories}

A (one dimensional, commutative) formal group law over a commutative ring $R$ is a formal power series $F(x, y)$ in $R[[x, y]]$ satisfying

$$
F(x, y)=F(y, x), \quad F(x, 0)=x, \quad F(x, F(y, z))=F(F(x, y), z) .
$$

Let $E^{*}(\cdot)$ be a complex oriented cohomology theory with base ring $R=E^{*}(\mathrm{pt})$. By [Qu71], this is equipped with a formal group law $F(x, y)$ over $R$, which expresses the corresponding first Chern class, denoted $c(\cdot)$, of a tensor product of two line bundles $\mathcal{L}_{1}$ and $\mathcal{L}_{2}$ on a space $X$ in terms of $c\left(\mathcal{L}_{1}\right)$ and $c\left(\mathcal{L}_{2}\right)$ :

$$
c\left(\mathcal{L}_{1} \otimes \mathcal{L}_{2}\right)=F\left(c\left(\mathcal{L}_{1}\right), c\left(\mathcal{L}_{2}\right)\right) .
$$

The reciprocal of Quillen's statement is false: there are formal group laws which do not come from complex oriented cohomology theories. However, if one translates and extends the axiomatics of oriented theories into the algebraic context, then to any formal group law one can associate the respective algebraic oriented cohomology theory [LM07]. In this paper we work in the more general algebraic setting, so $E^{*}(\cdot)$ stands for the respective algebraic oriented cohomology.

We refer to the (finite type) generalized flag variety $G / B$, and we let $T$ be the corresponding maximal torus. We use freely the corresponding root system terminology. As usual, we denote the set of roots by 
$\Phi$, the subsets of positive and negative roots by $\Phi^{+}$and $\Phi^{-}$, the simple roots and corresponding simple reflections by $\alpha_{i}$ and $s_{i}$ (for $i=1, \ldots, n$, where $n$ is the rank of the root system), the lattice of integral weights by $\Lambda$, the Weyl group by $W$, its longest element by $w_{\circ}$, and its strong Bruhat order by $\leq$. For each $w \in W$, we have the corresponding Schubert variety $X(w):=\overline{B w B}$. Given an arbitrary weight $\lambda$, let $\mathcal{L}_{\lambda}$ be the corresponding line bundle over $G / B$, that is, $\mathcal{L}_{\lambda}:=G \times_{B} \mathbb{C}_{-\lambda}$, where $B$ acts on $G$ by right multiplication, and the $B$-action on $\mathbb{C}_{-\lambda}=\mathbb{C}$ corresponds to the character determined by $-\lambda$.

We now consider the respective $T$-equivariant cohomology $E_{T}^{*}(\cdot)$ of spaces with a $T$-action, see e.g. [HHH05]. Its base ring $E_{T}^{*}(\mathrm{pt})$ can be identified (after completion) with the formal group algebra

$$
S:=R\left[\left[y_{\lambda}\right]\right]_{\lambda \in \Lambda} /\left(y_{0}, y_{\lambda+\nu}-F\left(y_{\lambda}, y_{\nu}\right)\right)
$$

of [CPZ13, Def. 2.4]. The Weyl group acts on $S$ by $w\left(y_{\lambda}\right):=y_{w \lambda}$.

The universal formal group law corresponds to complex cobordism. In this paper we focus on elliptic cohomology, denoted $E l l^{*}(\cdot)$, whose corresponding formal group law is that of an elliptic curve [MR07]. More precisely, for an elliptic curve given in Tate coordinates by $\left(1-\mu_{1} t-\mu_{2} t^{2}\right) s=t^{3}$, it is known that the respective formal group law is the hyperbolic one

$$
F_{h}(x, y)=\frac{x+y-\mu_{1} x y}{1+\mu_{2} x y}, \quad \text { defined over } R=\mathbb{Z}\left[\mu_{1}, \mu_{2}\right] .
$$

The hyperbolic formal group law is, in fact, a very natural one from a topological perspective. It implicitly appeared in Hirzebruch's celebrated book [Hi95] devoted to the proof of the Riemann-Roch theorem (cf. his earlier work). Indeed, the book is centered on the notion of a "(2-parameter) virtual generalized Todd genus". It turns out that the associated formal group law (via the one-to-one correspondence between genera and formal group laws) is precisely the one in (4).

Let us also mention some important special cases. In the trivial case $\mu_{1}=\mu_{2}=0, F_{h}(x, y)=F_{a}(x, y)$ is the additive group law, which corresponds to ordinary cohomology $H^{*}(\cdot)$. If $\mu_{2}=0$, and $\mu_{1}=1$, resp. $\mu_{1}$ is not specialized, then $F_{h}(x, y)=F_{m}(x, y)$ is the corresponding version of the multiplicative formal group law, associated to $K$-theory, and connective $K$-theory, respectively.

\subsection{Schubert and Bott-Samelson classes}

Given a Weyl group element $w$, consider a reduced word $I_{w}=\left(i_{1}, \ldots, i_{l}\right)$ for it, so $w=s_{i_{1}} \ldots s_{i_{l}}$. There is a Bott-Samelson resolution of the corresponding Schubert variety $X(w)$, which we denote by $\gamma_{I_{w}}: \Gamma_{I_{w}} \rightarrow X(w) \hookrightarrow G / B$. This determines a so-called Bott-Samelson class in $E_{T}^{*}(G / B)$ via the corresponding pushforward map, namely $\left(\gamma_{I_{w}}\right)_{!}(1)$. Here we let

$$
\zeta_{I_{w}}:=\left(\gamma_{I_{w}^{-1}}\right) !(1)
$$

where $I_{w}^{-1}:=\left(i_{l}, \ldots, i_{1}\right)$ is a reduced word for $w^{-1}$; we use $I_{w^{-1}}$, rather than $I_{w}$, in order to simplify the correspondence with the setup in [Bi99, Wil04], which is used in Sections 4 and 5 Note that $\zeta_{\emptyset}$ is the class of a point (where $\emptyset$ denotes the reduced word for the identity).

It is well-known that the Bott-Samelson classes are independent of the corresponding reduced words only for cohomology and $K$-theories (we can say that connective $K$-theory is the "last" case when this happens). In these cases, the Bott-Samelson classes are the Schubert classes, and they form bases of $H_{T}^{*}(G / B)$ and $K_{T}(G / B)$ over the corresponding formal group algebra $S$, as $w$ ranges over $W$. (More 
precisely, the Schubert classes in cohomology are the Poincaré duals to the fundamental classes of Schubert varieties, whereas in $K$-theory they are the classes of structure sheaves of Schubert varieties.) More generally, an important result in generalized cohomology Schubert calculus says that, by fixing a reduced word $I_{w}$ for each $w$, the Bott-Samelson classes $\left\{\zeta_{I_{w}}: w \in W\right\}$ form an $S$-basis of $E_{T}^{*}(G / B)$.

\subsection{The formal Demazure algebra}

Following [HMSZ13, §6] and [CZZ12, §3], consider the localization $Q$ of $S$ along all $x_{\alpha}$, for $\alpha \in \Phi$ (note the change of notation, from $y_{\lambda}$ to $x_{\lambda}$, cf. the convention at the beginning of Section 3.1), and define the twisted group algebra $Q_{W}$ to be the smash product $Q \# R[W]$, see [HMSZ13, Def. 6.1]. More precisely, as an $R$-module, $Q_{W}$ is $Q \otimes_{R} R[W]$, while the multiplication is given by

$$
q \delta_{w} \cdot q^{\prime} \delta_{w^{\prime}}=q\left(w q^{\prime}\right) \delta_{w w^{\prime}}, \quad q, q^{\prime} \in Q, w, w^{\prime} \in W .
$$

We denote $\delta_{i}:=\delta_{s_{i}}, x_{ \pm i}:=x_{ \pm \alpha_{i}}$, and $x_{ \pm i \pm j}:=x_{ \pm \alpha_{i} \pm \alpha_{j}}$, for $i, j \in\{1, \ldots, n\}$; similarly for the $y$ variables (to be used later). Following [HMSZ13, Def. 6.2] and [CZZ13], we define in $Q_{W}$ :

$$
X_{i}:=\frac{1}{x_{i}} \delta_{i}-\frac{1}{x_{i}}=\frac{1}{x_{i}}\left(\delta_{i}-1\right), \quad Y_{i}:=X_{i}+\kappa_{i}=\frac{1}{x_{-i}}+\frac{1}{x_{i}} \delta_{i}=\left(1+\delta_{i}\right) \frac{1}{x_{-i}},
$$

where $\kappa_{i}:=\frac{1}{x_{-i}}+\frac{1}{x_{i}}$. We call $X_{i}$ and $Y_{i}$ the Demazure and the push-pull element, respectively. The $R$-subalgebra $\mathbf{D}_{F}$ of $Q_{W}$ generated by the elements of $S$ and the elements $\left\{X_{i}\right\}$, or $\left\{Y_{i}\right\}$, is called the formal affine Demazure algebra. Its dual $\mathbf{D}_{F}^{\star}$ serves as an integral model for $E_{T}^{*}(G / B)$ [CZZ14].

Relations in the algebra $\mathbf{D}_{F}$ were given in [HMSZ13, Thm. 6.14] and [HMSZ13, Prop. 8.10]. In the case of the hyperbolic formal group law, these relations are:

(a) For all $i$, we have

$$
Y_{i}^{2}=\mu_{1} Y_{i}
$$

(b) If $\left\langle\alpha_{i}, \alpha_{j}^{\vee}\right\rangle=0$, so that $m_{i j}=2$, where $m_{i j}$ be the order of $s_{i} s_{j}$ in $W$, then

$$
Y_{i} Y_{j}=Y_{j} Y_{i} .
$$

(c) If $\left\langle\alpha_{i}, \alpha_{j}^{\vee}\right\rangle=\left\langle\alpha_{j}, \alpha_{i}^{\vee}\right\rangle=-1$, so that $m_{i j}=3$, then

$$
Y_{i} Y_{j} Y_{i}-Y_{j} Y_{i} Y_{j}=\mu_{2}\left(Y_{j}-Y_{i}\right) \text {. }
$$

There are also more involved relations if $m_{i j}=4$ or $m_{i j}=6$, given in [HMSZ13, Prop. 6.8]. These relations as well as (10) are called twisted braid relations. In the case of ordinary cohomology and $K$ theory, the twisted braid relations are the usual braid relations (since $\mu_{2}=0$ ).

Given a reduced word $I_{w}=\left(i_{1}, \ldots, i_{l}\right)$ for $w \in W$, define $X_{I_{w}}:=X_{i_{1}} \ldots X_{i_{l}}$ and $Y_{I_{w}}:=Y_{i_{1}} \ldots Y_{i_{l}}$. By [CZZ13], if we fix a reduced word $I_{w}$ for each $w \in W$, then $\left\{X_{I_{w}}: w \in W\right\}$ and $\left\{Y_{I_{w}}: w \in W\right\}$ are bases of the free left $Q$-module $Q_{W}$. Note that, in cohomology and $K$-theory, $X_{I_{w}}$ and $Y_{I_{w}}$ do not depend on the choice of the reduced word $I_{w}$, so we can simply write $X_{w}$ and $Y_{w}$.

A fundamental result in generalized cohomology Schubert calculus states that the Bott-Samelson classes $\zeta_{I_{w}}$, for $I_{w}=\left(i_{1}, \ldots, i_{l}\right)$, can be calculated recursively as follows (via the usual action of $\mathbf{D}_{F}$ on the Borel model of $E_{T}^{*}(G / B)$, see e.g. [GR13]):

$$
\zeta_{I_{w}}=Y_{i_{l}} \ldots Y_{i_{1}} \zeta_{\emptyset}
$$


By analogy with [11, we define the following classes:

$$
\widetilde{\zeta}_{I_{w}}:=X_{i_{l}} \ldots X_{i_{1}} \widetilde{\zeta}_{\emptyset}
$$

where $\widetilde{\zeta}_{\emptyset}=\zeta_{\emptyset}$ is the class of a point, and $I_{w}=\left(i_{1}, \ldots, i_{l}\right)$ is a reduced word for $w$, as above. Clearly, in ordinary cohomology we have $\zeta_{w}=\widetilde{\zeta}_{w}$, because $X_{i}=Y_{i}$ (and there is no dependence on $I_{w}$ ). In $K$-theory we have $X_{i}=Y_{i}-1$; for the geometric significance of the corresponding classes $\widetilde{\zeta}_{w}$, we refer to Remark 4.7

\subsection{The GKM model of equivariant cohomology}

In the GKM model, see e.g. [GKM98, GR13, HHH05, CPZ13], we embed $E_{T}^{*}(G / B)$ into $\bigoplus_{w \in W} S$, with pointwise multiplication. This comes from the embedding

$$
\begin{aligned}
& i^{*}: E_{T}^{*}(G / B) \rightarrow \bigoplus_{w \in W} E_{T}^{*}(\mathrm{pt}) \simeq \bigoplus_{w \in W} S, \quad \text { where } \\
& i^{*}:=\bigoplus_{w \in W} i_{w}^{*}, \quad \text { and } \quad i_{w}: \mathrm{pt} \rightarrow G / B, \text { with pt } \mapsto w^{-1} .
\end{aligned}
$$

There is a characterization of the image of this embedding, see e.g. [GR13]. We denote the elements of $\bigoplus_{w \in W} S$ by $\left(f_{w}\right)_{w \in W}$; alternatively, we view them as functions $f: W \rightarrow S$.

Finally, note that the algebras $Q_{W}$ and $\mathbf{D}_{F}$ act on the GKM model of $E_{T}^{*}(G / B)$, cf. [GR13, Eq. (3.19), (3.20)]. Using this action, we can calculate the classes $\zeta_{I_{w}}$ and $\widetilde{\zeta}_{I_{w}}$ recursively via (11) and (12), as the $\operatorname{class} \zeta_{\emptyset}=\widetilde{\zeta}_{\emptyset}$ is known, see [GR13, Eq. (4.4)].

\section{Formal root polynomials and their properties}

In this section we define formal root polynomials by extending the definitions in [Bi99, Gr02, Wil04], and begin their study. The setup is the one above, so it corresponds to an arbitrary formal group law.

\subsection{Definition and basic facts}

Consider the ring $Q_{W}[[\Lambda]]_{F}=S \otimes_{R} Q_{W}$, where the elements of $S$ on the left (denoted by $y$ 's) commute with the elements of $Q_{W}$. The formal root polynomials are elements of this ring which depend on a reduced word $I_{w}=\left(i_{1}, \ldots, i_{l}\right)$ for a Weyl group element $w$. In fact, we will define two formal root polynomials, corresponding to the Demazure and push-pull operators $X_{i}$ and $Y_{i}$ in (7). It is well-known that $I_{w}$ induces a so-called reflection order on the roots in $\Phi^{+} \cap w \Phi^{-}$, namely

$$
\Phi^{+} \cap w \Phi^{-}=\left\{\alpha_{i_{1}}, s_{i_{1}} \alpha_{i_{2}}, \ldots, s_{i_{1}} \ldots s_{i_{l-1}} \alpha_{i_{l}}\right\} .
$$

Definition 3.1. The formal $Y$-root polynomial corresponding to $I_{w}$ is

$$
\mathcal{R}_{I_{w}}^{Y}:=\prod_{k=1}^{l} h_{i_{k}}^{Y}\left(s_{i_{1}} \ldots s_{i_{k-1}} \alpha_{i_{k}}\right), \quad \text { where } h_{i}^{Y}(\lambda):=1-y_{\lambda} Y_{i} .
$$

Similarly, we define the formal $X$-root polynomial by replacing $h_{i}^{Y}(\lambda)$ with $h_{i}^{X}(\lambda):=1+y_{-\lambda} X_{i}$. 
Example 3.2. For type $A_{2}$ and $w=s_{i} s_{j} s_{i}, I_{w}=(i, j, i)$, we obtain (cf. the notation in Section 2.3)

$$
\begin{aligned}
\mathcal{R}_{(i, j, i)}^{Y} & =\left(1-y_{i} Y_{i}\right)\left(1-y_{i+j} Y_{j}\right)\left(1-y_{j} Y_{i}\right) \\
& =-y_{i} y_{i+j} y_{j} Y_{i} Y_{j} Y_{i}+y_{i} y_{i+j} Y_{i} Y_{j}+y_{j} y_{i+j} Y_{j} Y_{i}+\left(\kappa_{i} y_{i} y_{j}-y_{i}-y_{j}\right) Y_{i}-y_{i+j} Y_{j}+1
\end{aligned}
$$

Consider a ring homomorphism ev : $S \otimes_{R} Q \rightarrow Q$ given by $y_{\lambda} \mapsto x_{-\lambda}$, for $\lambda \in \Lambda$. Since $Q_{W}[[\Lambda]]_{F}$ is a left $S \otimes_{R} Q$-module, it induces a homomorphism of left $S \otimes_{R} Q$-modules ev: $Q_{W}[[\Lambda]]_{F} \rightarrow Q_{W}$, which we call the evaluation map.

Lemma 3.3. We have

$$
\operatorname{ev}\left(\mathcal{R}_{I_{w}}^{X}\right)=\delta_{w}, \quad \operatorname{ev}\left(\mathcal{R}_{I_{w}}^{Y}\right)=\theta_{I_{w}} \delta_{w}, \quad \text { where } \theta_{I_{w}}:=\prod_{k=1}^{l} \theta\left(s_{i_{1}} \ldots s_{i_{k-1}} \alpha_{i_{k}}\right), \quad \theta(\lambda)=\frac{-x_{-\lambda}}{x_{\lambda}} \in S
$$

Furthermore, $\theta_{I_{w}}$ does not depend on the choice of the reduced word $I_{w}$ for $w$, so we write $\theta_{w}=\theta_{I_{w}}$.

We will call $\theta_{w}$ the normalizing parameter.

Remark 3.4. In the ordinary cohomology case, the formal root polynomials coincide with those Billey [Bi99, Definition 2], and we have $\theta(\lambda)=1$. In the $K$-theory case, the $Y$-root polynomials coincide with those of Willems [Wil04, Section 5], and we have $\theta_{w}=e^{\rho-w \rho}$, where $\rho=\frac{1}{2} \sum_{\alpha \in \Phi^{+}} \alpha$, as usual.

\subsection{Independence of choices of reduced words}

We now provide necessary and sufficient conditions for the root polynomials $\mathcal{R}_{I_{w}}^{Y}$ and $\mathcal{R}_{I_{w}}^{X}$ to be independent of a choice of $I_{w}$, hence generalizing the respective results of Billey [Bi99] and Willems [Wil04]. For simplicity, we refer here only to the $Y$-root polynomial, and let $h_{i}=h_{i}^{Y}$.

Proposition 3.5. If $\left\langle\alpha_{i}, \alpha_{j}^{\vee}\right\rangle=0$, i.e., $m_{i j}=2$, then the $h_{i}$ 's satisfy the commuting relations for all $\lambda, \nu \in \Lambda$, that is, $h_{i}(\lambda) h_{j}(\nu)=h_{j}(\nu) h_{i}(\lambda)$.

Proposition 3.6. Consider a root system containing a pair of simple roots $\alpha_{i}, \alpha_{j}$ with $m_{i j}=3$, which we fix. Also assume that 2 is not a zero divisor in the coefficient ring $R$. Then the following are equivalent.

(a) The underlying formal group law is the hyperbolic one (4).

(b) We have

$$
h_{i}(\lambda) h_{j}(\lambda+\nu) h_{i}(\nu)=h_{j}(\nu) h_{i}(\lambda+\nu) h_{j}(\lambda)
$$

for all $\lambda, \nu \in \Lambda$. We say that $h_{i}, h_{j}$ satisfy the (type A) Yang-Baxter relation for all weights.

(c) We have

$$
h_{i}\left(\alpha_{i}\right) h_{j}\left(\alpha_{i}+\alpha_{j}\right) h_{i}\left(\alpha_{j}\right)=h_{j}\left(\alpha_{j}\right) h_{i}\left(\alpha_{i}+\alpha_{j}\right) h_{j}\left(\alpha_{i}\right) .
$$

We say that $h_{i}, h_{j}$ satisfy the (type A) Yang-Baxter relation for the corresponding simple roots.

Now recall from [Bi99, Wil04] the Yang-Baxter relations of types $B$ and $G$.

Proposition 3.7. Consider a root system containing a pair of simple roots $\alpha_{i}, \alpha_{j}$ with $m_{i j}=4$ or $m_{i j}=$ 6, which we fix. Also assume that the underlying formal group law is the hyperbolic one. Then $h_{i}$ and $h_{j}$ satisfy the (type B, resp. type $G$ ) Yang-Baxter relation for all weights, cf. (16). 
Based on Propositions 3.5, 3.6, 3.7, we deduce the following result.

Theorem 3.8. The root polynomial $\mathcal{R}_{I_{w}}^{Y}$ does not depend on the choice of $I_{w}$ if the underlying formal group law $F(x, y)$ is the hyperbolic one; so we can write $\mathcal{R}_{w}^{Y}$ instead. The reciprocal holds if the corresponding root system contains a pair of simple roots $\alpha_{i}, \alpha_{j}$ with $m_{i j}=3$.

Remarks 3.9. (1) The first part of Theorem 3.8 appears as [Bi99, Theorem 2], resp. [Wil04, Theorem 5.2], in the case of the additive formal group law, resp. the multiplicative one.

(2) An alternative proof of the first part of Theorem 3.8, which is uniform for all Lie types, is given in Section 4.1 .

\section{Applications of formal root polynomials in the hyperbolic case}

\subsection{The coefficients of formal root polynomials}

In this section we present our main application of formal root polynomials in the hyperbolic case, which is continued in the next section.

We start with an arbitrary formal group law $F(x, y)$. Consider the root polynomials $\mathcal{R}_{I_{w}}^{Y}$ and $\mathcal{R}_{I_{w}}^{X}$ as elements of $Q_{W}[[\Lambda]]_{F}$. Recall that, after we fix a reduced word $I_{v}$ for each $v \in W$, the elements $\left\{Y_{I_{v}}: v \in W\right\}$ and $\left\{X_{I_{v}}: v \in W\right\}$ form bases of the left $S \otimes_{R} Q$-module $Q_{W}[[\Lambda]]_{F}$.

Definition 4.1. Let $K^{Y}\left(I_{v}, I_{w}\right)$, resp. $K^{X}\left(I_{v}, I_{w}\right)$, denote the coefficient of $Y_{I_{v}}$ in the expansion of $\mathcal{R}_{I_{w}}^{Y}$, resp. the coefficient of $X_{I_{v}}$ in the expansion of $\mathcal{R}_{I_{w}}^{X}$, i.e.

$$
\mathcal{R}_{I_{w}}^{Y}=\sum_{v \leq w} K^{Y}\left(I_{v}, I_{w}\right) Y_{I_{v}}, \quad \mathcal{R}_{I_{w}}^{X}=\sum_{v \leq w} K^{X}\left(I_{v}, I_{w}\right) X_{I_{v}}
$$

By analogy with the setup of [Bi99], we call $K^{Y}\left(I_{v}, I_{w}\right)$ and $K^{X}\left(I_{v}, I_{w}\right)$ (normalized) formal Kostant polynomials.

From now on, we assume that we are in the hyperbolic case, so by Theorem 3.8 we know that the root polynomials do not depend on the choice of a reduced word. However, we will not use this knowledge in this section, so we will continue to use the notation $\mathcal{R}_{I_{w}}^{Y}$ and $\mathcal{R}_{I_{w}}^{X}$.

Observe that the free left $Q$-module $Q_{W}$ also has a basis $\left\{\delta_{w}: w \in W\right\}$. Consider the following expansions, cf. [CZZ13, Lemmas 3.1 and 3.2]:

$$
\delta_{w}=\sum_{v \leq w} b_{w, I_{v}}^{Y} Y_{I_{v}}, \quad \delta_{w}=\sum_{v \leq w} b_{w, I_{v}}^{X} X_{I_{v}}
$$

for some coefficients $b_{w, I_{v}}^{Y}$ and $b_{w, I_{v}}^{X}$ in $S$ (in $x$ 's).

By applying the evaluation map ev to (17) and by using Lemma 3.3, we derive the following result. Here we use the sign change involution $*$ on the formal group algebra $S$, given by $y_{\lambda} \mapsto y_{-\lambda}$ (not to be confused with ev).

Theorem 4.2. In the hyperbolic case, we have in $S$ :

$$
b_{w, I_{v}}^{Y}=*\left(\theta_{w} K^{Y}\left(I_{v}, I_{w}\right)\right), \quad b_{w, I_{v}}^{X}=*\left(K^{X}\left(I_{v}, I_{w}\right)\right) .
$$

In particular, $K^{Y}\left(I_{v}, I_{w}\right)$ (resp. $K^{X}\left(I_{v}, I_{w}\right)$ ), and hence $\mathcal{R}_{I_{w}}^{Y}$ (resp. $\mathcal{R}_{I_{w}}^{X}$ ), do not depend on the choice of $I_{w}$, so we can use the notation $K^{Y}\left(I_{v}, w\right)$ and $\mathcal{R}_{w}^{Y}\left(\right.$ resp. $K^{X}\left(I_{v}, w\right)$ and $\left.\mathcal{R}_{w}^{X}\right)$ in this case. 
Remarks 4.3. (1) The formula in Theorem 4.2 relates the coefficients of the $Y$-root polynomial with the entries of the transformation matrix between two natural bases of the twisted formal group algebra; similarly for the $X$-root polynomial. This provides an efficient way to compute these matrices, see Example 4.4. For cohomology and $K$-theory, these matrices feature prominently in the work of Kostant-Kumar [KK90], as they encode information about the singularities of Schubert varieties; see [BL00, Chapter 7].

(2) Theorem 4.2 contains an alternative, type-independent proof to the first part of Theorem 3.8 .

Example 4.4. Consider the root system $A_{2}$ and the Lorentz formal group law, i.e., (4) with $\mu_{1}=0$. For each permutation $v \neq w_{\circ}$ in $S_{3}$ we have a unique reduced word, and choose $I_{w_{\circ}}=(1,2,1)$. Based on Example 3.2. while also noting that here $\kappa_{i}=0, \theta_{w}=1$ (cf. Remark 3.4), and $y_{-\alpha}=-y_{\alpha}$, we have

$$
\delta_{w_{\circ}}=y_{1} y_{1+2} y_{2} Y_{1} Y_{2} Y_{1}+y_{1} y_{1+2} Y_{1} Y_{2}+y_{2} y_{1+2} Y_{2} Y_{1}+\left(y_{1}+y_{2}\right) Y_{1}+y_{1+2} Y_{2}+1 \text {. }
$$

If we choose $I_{w_{\circ}}=(2,1,2)$ instead, we need to adjust the above expansion by rewriting $Y_{1} Y_{2} Y_{1}$ as $Y_{2} Y_{1} Y_{2}+\mu_{2}\left(Y_{2}-Y_{1}\right)$ via (10), and then collecting terms. Alternatively, we can avoid using the twisted braid relation by using the expression for $\mathcal{R}_{(2,1,2)}^{Y}$ in Example 3.2. Note that, although $\mathcal{R}_{(1,2,1)}^{Y}=\mathcal{R}_{(2,1,2)}^{Y}$, the expressions for them given in Example 3.2 are different.

Let us now turn to a generalization of the cohomology and $K$-theory Schubert structure constants. Given a choice of reduced words $I_{w}$, let $Y_{I_{w}}^{*}$ in the dual Demazure algebra $\mathbf{D}_{F}^{\star}$ be the usual dual of $Y_{I_{w}}$, see Section 2.3, these are not the Bott-Samelson classes in general (see Section 5]. Let

$$
Y_{I_{u}}^{*} \cdot Y_{I_{v}}^{*}=\sum_{w \geq u, v} p_{I_{u}, I_{v}}^{I_{w}} Y_{I_{w}}^{*}
$$

be the expansion of the given product into the basis $\left\{Y_{I_{w}}^{*}\right\}$ of $\mathbf{D}_{F}^{\star}$. We follow the approach in Billey's paper [Bi99] related to the cohomology structure constants. We start by generalizing [Bi99, Lemma 5.3]. Proposition 4.5. The coefficients $p_{I_{u}, I_{v}}^{I_{w}}$ can be computed recursively by

$$
p_{I_{u}, I_{v}}^{I_{w}}=\frac{1}{b_{w, I_{w}}^{Y}}\left(b_{w, I_{u}}^{Y} b_{w, I_{v}}^{Y}-\sum_{t<w} p_{I_{u}, I_{v}}^{I_{t}} b_{w, I_{t}}^{Y}\right)
$$

starting with $w=u\left(\right.$ for $\left.v \leq u, p_{I_{u}, I_{v}}^{I_{u}}=b_{u, I_{v}}^{Y}\right)$ and going up in length.

Corollary 4.6. For any formal group law, the coefficients $p_{I_{u}, I_{v}}^{I_{w}}$ do not depend on the choice of $I_{w}$, so we can write $p_{I_{u}, I_{v}}^{w}=p_{I_{u}, I_{v}}^{I_{w}}$.

Hence, in the hyperbolic case, via Theorem 4.2, we are able to compute $p_{I_{u}, I_{v}}^{w}$ in terms of the coefficients $K^{Y}\left(I_{v}, w\right)$ of $Y$-root polynomials. This gives another application of these polynomials. We have completely similar results for the multiplication of the basis elements $\left\{X_{I_{w}}^{*}\right\}$.

\subsection{Localization formulas in connective $K$-theory}

We work in the setup of connective $K$-theory, whose formal group law is obtained by setting $t:=\mu_{1}$ and $\mu_{2}=0$ in (4), cf. Section 2.1. Recall from Section 2.3 that the corresponding operators $Y_{i}$ and $X_{i}$ satisfy $Y_{i}^{2}=t Y_{i}, X_{i}^{2}=-t X_{i}$, and the usual braid relations. We define the functions

$$
\psi^{v}:=\left(\widetilde{\zeta}_{v w_{\circ}}\right)^{\circ}, \quad \xi^{v}:=\left(\zeta_{v w_{\circ}}\right)^{\circ}
$$

where, given a function $f: W \rightarrow S$, we define $f^{\circ}: W \rightarrow S$ by $f^{\circ}(w):=f\left(w w_{\circ}\right)$. 
Remark 4.7. Consider ordinary $K$-theory, so $t=1$. The evaluation $\xi^{v}(w)$ can be interpreted geometrically as $i_{w w_{\circ}}^{*}\left(\left[\mathcal{O}_{v w_{\circ}}\right]\right)$, where $\mathcal{O}_{u}$ is the structure sheaf of the Schubert variety indexed by $u$ (see Section 2.4). The Poincaré dual of the $K$-theory class $\zeta_{v}=\left[\mathcal{O}_{v}\right]$ is the class of the ideal sheaf of the Schubert variety indexed by $v$, which under $i_{w}^{*}$ is mapped to $* \psi^{v}(w)$, see e.g. [KK90], [GK08, Prop. 2.1]. The duality property, which holds for both cohomology and $K$-theory, says that there is a non-degenerate pairing

$$
p_{!}\left(\zeta_{v} \cdot * \psi^{w}\right)=p_{!}\left(Y_{v^{-1}} \zeta_{\emptyset} \cdot X_{\left(w_{\circ} v\right)^{-1}}\left(* \psi^{w_{\circ}}\right)\right)=\delta_{v, w}
$$

where $p_{\text {! }}$ is the degree map for cohomology and the Euler characteristic for $K$-theory.

The following formulas generalize the ones of Billey [Bi99], in cohomology, and Graham-Willems [Gr02, Wil04], in ordinary $K$-theory. They are proved by induction of $\ell(v)$. To state our result, consider a reduced word $I_{w}=\left(i_{1}, \ldots, i_{l}\right)$ for $w$, and recall the corresponding reflection order [15] on $\Phi^{+} \cap w \Phi^{-}$, namely $\left(\beta_{1}, \ldots, \beta_{l}\right)$ with $\beta_{j}:=s_{i_{1}} \ldots s_{i_{j-1}} \alpha_{i_{j}}$.

Theorem 4.8. In connective $K$-theory, we have $\psi^{v}(w)=\theta_{w} K^{Y}(v, w)$. More explicitly, we have

$$
\psi^{v}(w)=\theta_{w} \sum_{1 \leq j_{1}<\ldots<j_{k} \leq l}(-1)^{k} t^{k-\ell(v)} y_{\beta_{j_{1}}} \ldots y_{\beta_{j_{k}}},
$$

where $\ell(v) \leq k \leq l$, and the summation ranges over the integer sequences $1 \leq j_{1}<\ldots<j_{k} \leq l$ for which the corresponding subword $\left(p_{1}, \ldots, p_{k}\right)=\left(i_{j_{1}}, \ldots, i_{j_{k}}\right)$ of $I_{w}$ is subject to $Y_{p_{1}} \ldots Y_{p_{k}}=$ $t^{k-\ell(v)} Y_{v}$. Similar results hold for $\xi^{v}$.

\subsection{The results of Billey and Graham-Willems revisited; duality in connective $K$-theory}

The following result is an immediate consequence of Theorem 4.2 and the duality result for an arbitrary cohomology theory in [CZZ13, Theorem 12.4].

Theorem 4.9. The connective $K$-theory analogue of the duality (20) is equivalent to both formulas, for $\psi^{v}$ and $\xi^{v}$, in Theorem 4.8

Let us comment on this result. Based on the duality result 20 in cohomology and $K$-theory, we have a new proof of Theorem 4.8 in these cases, and hence of the formulas of Billey and Graham-Willems. This proof has the advantage of being very short, completely transparent, and uniform (in all Lie types and for both cohomology and $K$-theory). On the other hand, by reversing our reasoning, in connective $K$-theory we can rely on the proof of Theorem 4.8, and derive the analogue of the duality [20], which seems new.

\section{Bott-Samelson classes in the hyperbolic case}

In the previous section, we have considered two families of elements in $E_{T}^{*}(G / B)$, namely the BottSamelson classes $\zeta_{I_{v}}$ and $X_{I_{v}}^{*}$, both of which are viewed as functions from $W$ to $S$ (recall that $X_{I_{v}}^{*}$ are evaluated at $\delta_{w}$, cf. the expansion (18)). It is not hard to see (by the duality argument in Section 4.3) that these families essentially concide in $K$-theory, but not beyond that, due to the dependence on the reduced word $I_{v}$. Furthemore, in Theorem 4.2 we have been able to express $X_{I_{v}}^{*}\left(\delta_{w}\right)$ in the hyperbolic case based on formal root polynomials, thus generalizing the results of Billey and Willems in ordinary cohomology and $K$-theory. We now refer to the calculation of $\zeta_{I_{v}}$ in the hyperbolic case, which turns out to be a 
considerably harder problem. For simplicity, we denote the parameters in (4) by $t:=\mu_{1}$ and $u:=-\mu_{2}$, so the underlying ring $R$ is $\mathbb{Z}[t, u]$.

Based on the analogy with [21], as well as experimental evidence, we formulate the following positivity conjecture. To state it, consider $v \leq w$, and let $\Phi^{+} \cap w \Phi^{-}=\left\{\beta_{1}, \ldots, \beta_{l}\right\}$; also let $z_{i}=y_{-\beta_{i}}$.

Conjecture 5.1. (1) If $t=0, \zeta_{I_{v w_{\circ}}}\left(w w_{\circ}\right)$ is a sum with positive coefficients of terms of the form

$$
u^{(m-\ell(v)) / 2} z_{j_{1}} \ldots z_{j_{m}},
$$

where $\ell(v) \leq m \leq l, m-\ell(v)$ is even, and $1 \leq j_{1}<\ldots<j_{m} \leq l$.

(2) If $t=1, \zeta_{I_{v w_{0}}}\left(w w_{\circ}\right)$ is a sum with positive coefficients of terms of the form

$$
(-1)^{k-\ell(v)} u^{(m-k) / 2} z_{j_{1}} \ldots z_{j_{m}},
$$

where $\ell(v) \leq k \leq m \leq l, m-k$ is even, and $1 \leq j_{1}<\ldots<j_{m} \leq l$.

If the conjecture is true, it would be interesting to find the geometric reason for positivity.

\section{A Schubert basis via the Kazhdan-Lusztig basis}

An important open problem in Schubert calculus beyond $K$-theory is defining a Schubert basis, i.e., classes which are independent of a reduced word for the indexing Weyl group elements. The standard topological approach works if the Schubert variety $X(w)$ is smooth, and the corresponding class $[X(w)]$ has a simple formula; namely, by [GR13][Eq. (5.3)], cf. also [BL00, Theorem 7.2.1], in the GKM model of $E_{T}^{*}(G / B)$ (discussed in Section 2.4, we have:

$$
[X(w)]_{v}=\frac{\prod_{\beta \in \Phi^{+}} y_{-\beta}}{\prod_{\substack{\beta \in \Phi^{+} \\ s_{\beta} v \leq w}} y_{-\beta}}
$$

for $v \leq w$ in the Weyl group $W$; otherwise $[X(w)]_{v}=0$.

In general, an axiomatic approach (called "combinatorial forcing") was suggested in [GR13]. We propose a different approach in equivariant elliptic cohomology, which uses the Kazhdan-Lusztig basis of the corresponding Hecke algebra, and provide support for it.

Instead of the usual generators $T_{i}$ of the Hecke algebra $\mathcal{H}$ of the Weyl group $W$, we use $\tau_{i}:=t T_{i}$, where $t=q^{-1 / 2}$. In (4) we set $\mu_{1}=1$ and $\mu_{2}=-\left(t+t^{-1}\right)^{-2}=-u$, so we let the base ring $R$ be $\mathbb{Z}\left[t^{ \pm 1},\left(t+t^{-1}\right)^{-1}\right]$. In [CZZ13, LNZ] it is shown that $\mathcal{H} \otimes_{\mathbb{Z}\left[t^{ \pm 1}\right]} R$ is isomorphic to the $R$-subalgebra of the corresponding formal Demazure algebra $\mathbf{D}_{F}$ (see Section 2.3) generated by the elements $Y_{i}$ only; the isomorphism is given by $\tau_{i} \mapsto\left(t+t^{-1}\right) Y_{i}-t$.

Consider the Kazhdan-Lusztig basis $\left\{\gamma_{w}: w \in W\right\}$ of $\mathcal{H}$, given by

$$
\gamma_{w}=\tau_{w}+\sum_{v<w} t \pi_{v w}(t) \tau_{v}
$$

where $\pi_{v w}(t)$ are the Kazhdan-Lusztig polynomials (in terms of the classical notation, we have $P_{v w}(t)=$ $\left.t^{-(\ell(w)-\ell(v)-1)} \pi_{v w}(t)\right)$. For $w \in W$, denote by $\Gamma_{w}$ the element of $\mathbf{D}_{F}$ which corresponds to $\gamma_{w}$ via the 
above isomorphism. We now define our Schubert classes, which we call Kazhdan-Lusztig (KL-) Schubert classes.

Definition 6.1. Consider the element $\mathfrak{S}_{w}$ in $E l l_{T}^{*}(G / B)$ given by $\left(t+t^{-1}\right)^{-\ell(w)} \Gamma_{w^{-1}}\left(\zeta_{\emptyset}\right)$ under the action of $\mathbf{D}_{F}$ on the GKM model of $E l l_{T}^{*}(G / B)$.

Theorem 6.2. The KL-Schubert classes $\left\{\mathfrak{S}_{w}: w \in W\right\}$ form a basis of $\operatorname{Ell}_{T}^{*}(G / B)$. In the limit $t \rightarrow 0$, the class $\mathfrak{S}_{w}$ becomes the Bott-Samelson class $\zeta_{w}$ in K-theory, which coincides with the corresponding Schubert class.

We conjecture the following natural property for the KL-Schubert classes $\mathfrak{S}_{w}$.

Conjecture 6.3. If the Schubert variety $X(w)$ is smooth, then the class $[X(w)]$ in [22] coincides with $\mathfrak{S}_{w}$.

We tested this conjecture on the computer. Moreover, we proved the special cases below.

Theorem 6.4. Conjecture 6.3 is true in the following cases (which all correspond to non-singular Schubert varieties):

(1) in all types for $w$ which is a product of distinct simple reflections;

(2) in all classical types $A_{n-1}, B_{n}, C_{n}, D_{n}$, for $w^{-1}$, where $w$ is a highest coset representative for $W_{n} / W_{n-1}$ (in particular, for $w=w_{\circ}$, the longest element, we have $\mathfrak{S}_{w_{\circ}}=1$ ).

Remark 6.5. Case (1) of Theorem 6.4 is easy, because the formal group law is not involved in the calculation of the respective classes. However, this no longer happens in case (2), which is therefore highly non-trivial.

\section{References}

[AJS94] H. Andersen, J. Jantzen, and W. Soergel. Representations of quantum groups at a $p$ th root of unity and of semisimple groups in characteristic $p$ : independence of $p$. Astérisque, 220, 1994.

[Bi99] S. Billey. Kostant polynomials and the cohomology ring for G/B. Duke J. Math., 96:205224, 1999.

[BL00] S. Billey and V. Lakshmibai. Singular loci of Schubert varieties. Progress in Mathematics 182, Birkhäuser Boston, 2000, xii+251 pp.

[CZZ12] B. Calmès, K. Zainoulline, and C. Zhong. A coproduct structure on the formal affine Demazure algebra. To appear in Math. Zeitschrift. arXiv:1209.1676.

[CZZ13] B. Calmès, K. Zainoulline, and C. Zhong. Push-pull operators on the formal affine Demazure algebra and its dual. arXiv:1312.0019, 2013.

[CZZ14] B. Calmès, K. Zainoulline, and C. Zhong. Equivariant oriented cohomology of flag varieties. arXiv:1409.7111, 2014.

[CPZ13] B. Calmès, V. Petrov, and K. Zainoulline. Invariants, torsion indices and oriented cohomology of complete flags. Ann. Sci. École Norm. Sup. (4), 46:405-448, 2013. 
[GR13] N. Ganter, A. Ram. Generalized Schubert calculus. J. Ramanujan Math. Soc., 28A:149-190, 2013.

[GKM98] M. Goresky, R. E. Kottwitz, and R. D. MacPherson. Equivariant cohomology, Koszul duality, and the localization theorem. Invent. Math., 131:25-83, 1998.

[Gr02] W. Graham. Equivariant $K$-theory and Schubert varieties. Preprint, 2002.

[GK08] W. Graham and S. Kumar. On positivity in $T$-equivariant $K$-theory of flag varieties. Int. Math. Res. Not., 2008, Article ID rnn093, 43 pp.

[HHH05] M. Harada, A. Henriques, and T. Holm. Computation of generalized equivariant cohomologies of Kac-Moody flag varieties. Adv. Math., 197:198-221, 2005.

[Hi95] F. Hirzebruch. Topological methods in algebraic geometry. Classics in Mathematics. Springer, Berlin, 1995. xii+234 pp.

[HMSZ13] A. Hoffnung, J. Malagón-López, A. Savage, and K. Zainoulline. Formal Hecke algebras and algebraic oriented cohomology theories. Selecta Math., 20:1247-1248, 2014.

[HK11] J. Hornbostel and V. Kiritchenko. Schubert calculus for algebraic cobordism. J. Reine Angew. Math., 656:59-85, 2011.

[KK13] V. Kiritchenko and A. Krishna. Equivariant cobordism of flag varieties and of symmetric varieties. Transform. Groups, 18:391-413, 2013.

[KK86] B. Kostant and S. Kumar. The nil Hecke ring and cohomology of $G / P$ for a Kac-Moody group. Adv. Math., 62:187-237, 1986.

[KK90] B. Kostant and S. Kumar. T-equivariant $K$-theory of generalized flag varieties. J. Diff. Geom. 32:549-603, 1990.

[LNZ] M.-A. Leclerc, E. Neher, and K. Zainoulline. Elliptic Demazure algebras for Kac-Moody root systems. Work in progress.

[LZ14] C. Lenart and K. Zainoulline. Towards generalized cohomology Schubert calculus via formal root polynomials. arXiv:1408.5952, 2014.

[LM07] M. Levine and F. Morel. Algebraic Cobordism. Springer Monographs in Mathematics. Springer, Berlin, 2007.

[MR07] Elliptic cohomology. Geometry, applications, and higher chromatic analogues. Edited by H. Miller and D. Ravenel. London Math. Soc. Lecture Note Ser., 342. Cambridge Univ. Press, Cambridge, 2007. xii+364 pp.

[Qu71] D. Quillen. Elementary proofs of some results of cobordism theory using Steenrod operations. Adv. Math., 7:29-56, 1971.

[Wil04] M. Willems. Cohomologie et $K$-théorie équivariantes des variétés de Bott-Samelson et des variétés de drapeaux. Bull. Soc. Math. France, 132:569-589, 2004. 